\title{
Aminolysis of Y-Substituted Phenyl X-Substituted Cinnamates and Benzoates: Effect of Modification of Nonleaving Group from Benzoyl to Cinnamyl
}

\author{
Ik-Hwan Um, ${ }^{*}$ Youn-Min Park, Mizue Fujio, ${ }^{\dagger}$ Masaaki Mishima, ${ }^{\dagger}$ and Yuho Tsuno ${ }^{\dagger}$
}

Division of Nano Sciences and Department of Chemistry, Ewha Womans University, Seoul $120-750$, Korea

Tel: 82-2-3277-2349; Fax: 82-2-3277-2844; E-mail: ihum@ewha.ac.kr

†Institute for Materials Chemistry and Engineering, Kyushu University, Hakozaki, Higashiku, Fukuoka 812-8581, Japan

\section{Table of Contents}

(1) Figure S1. Hammett plots for the reactions of 2,4-dinitrophenyl X-substituted cinnamates (1a-e) with piperidine $(\bullet)$, morpholine $(\mathbf{\bullet})$, and piperazinium ion $(\mathbf{\Lambda})$ in $80 \mathrm{~mol}$ $\% \mathrm{H} 2 \mathrm{O} / 20 \mathrm{~mol} \%$ DMSO at $25.0{ }^{\circ} \mathrm{C}$

(2) ${ }^{1} \mathrm{H}$ NMR spectra for 2,4-dinitrophenyl 4-nitrocinnamate (1a), 2,4-dinitrophenyl 4chlorocinnamate (1b), 2,4-dinitrophenyl 4-methylcinnamate (1d), 2,4-dinitrophenyl 4methoxycinnamate (1e), 4-formylphenyl cinnamate (3c), 4-(ethoxycarbonyl)phenyl cinnamate (3e), 3-chlorophenyl cinnamate (3f), 3-acetylphenyl cinnamate (3g) -- (S4 - S7) 
(3) Tables S1 - S24. Summary of the Kinetic Data for the Reactions of 1a-e with Secondary Amines in $80 \mathrm{~mol} \% \mathrm{H}_{2} \mathrm{O} / 20 \mathrm{~mol} \%$ DMSO at $25.0 \pm 0.1^{\circ} \mathrm{C}$. -------- (S8 - S19)

(4) Tables S25 - S38. Summary of the Kinetic Data for the Reactions of 3a-g with Secondary Amines in $80 \mathrm{~mol} \% \mathrm{H}_{2} \mathrm{O} / 20 \mathrm{~mol} \% \mathrm{DMSO}$ at $25.0 \pm 0.1^{\circ} \mathrm{C}$. ------- $(\mathrm{S} 20$ - S26)

(5) Table S39. Summary of the Kinetic Data for the Reactions of $\mathbf{4 e}$ with Piperidine in 80 $\mathrm{mol} \% \mathrm{H}_{2} \mathrm{O} / 20 \mathrm{~mol} \% \mathrm{DMSO}$ at $25.0 \pm 0.1^{\circ} \mathrm{C}$

(6) Tables S40 - S43. Summary of the Kinetic Data for the Reactions of 4a, 4c, 4d, 4e with Morpholine in $80 \mathrm{~mol} \% \mathrm{H}_{2} \mathrm{O} / 20 \mathrm{~mol} \%$ DMSO at $25.0 \pm 0.1^{\circ} \mathrm{C}$. $(\mathrm{S} 27-\mathrm{S} 29)$ 


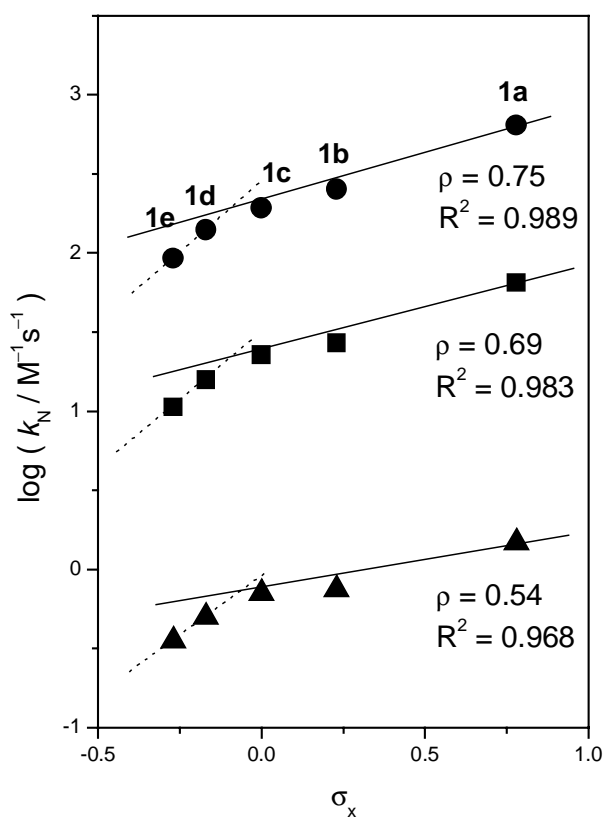

Figure S1. Hammett plots for the reactions of 2,4-dinitrophenyl X-substituted cinnamates (1a-e) with piperidine $(\bullet)$, morpholine $(\boldsymbol{\bullet})$, and piperazinium ion ( $\bullet$ ) in $80 \mathrm{~mol} \% \mathrm{H} 2 \mathrm{O} / 20$ mol \% DMSO at $25.0{ }^{\circ} \mathrm{C}$. 
${ }^{1} \mathrm{H}$ NMR spectrum for 2,4-dinitrophenyl 4-nitrocinnamate (1a)
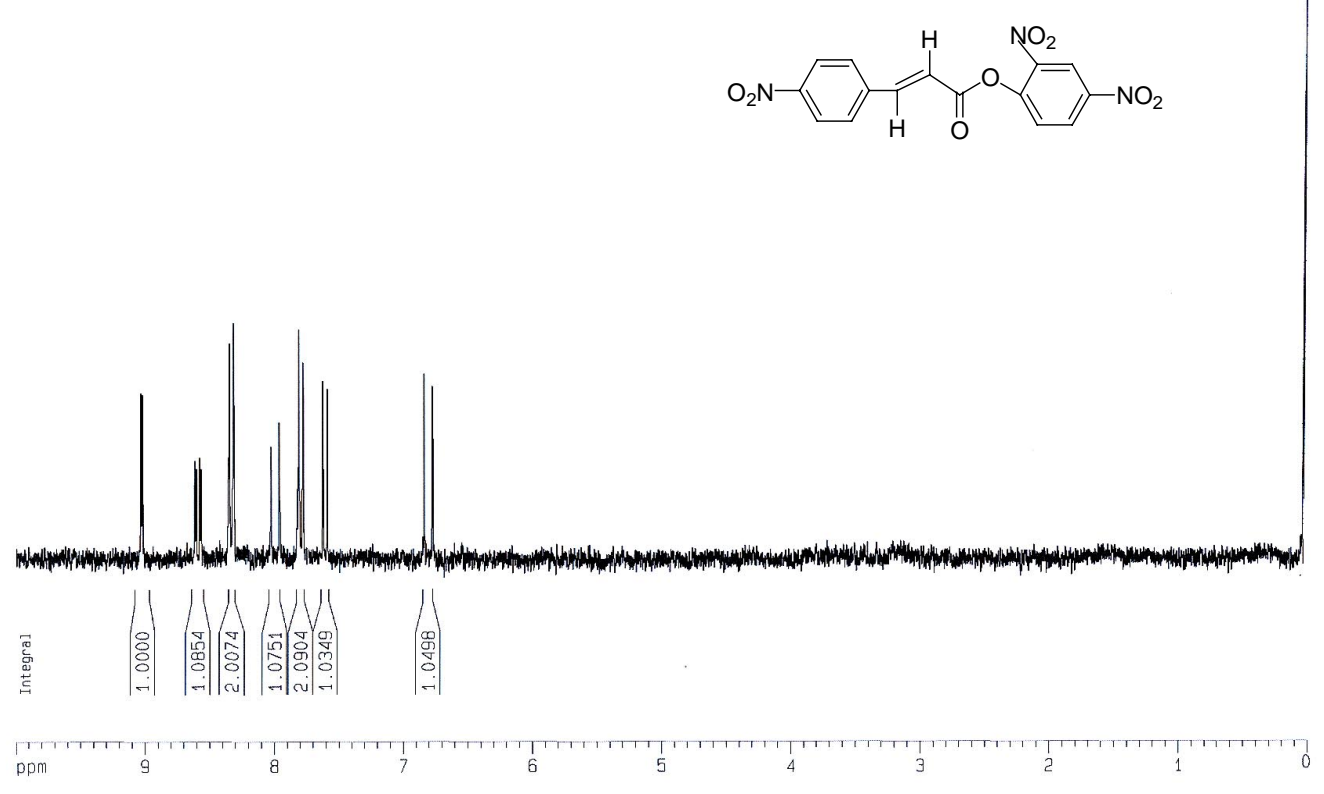

${ }^{1} \mathrm{H}$ NMR spectrum for 2,4-dinitrophenyl 4-chlorocinnamate (1b)

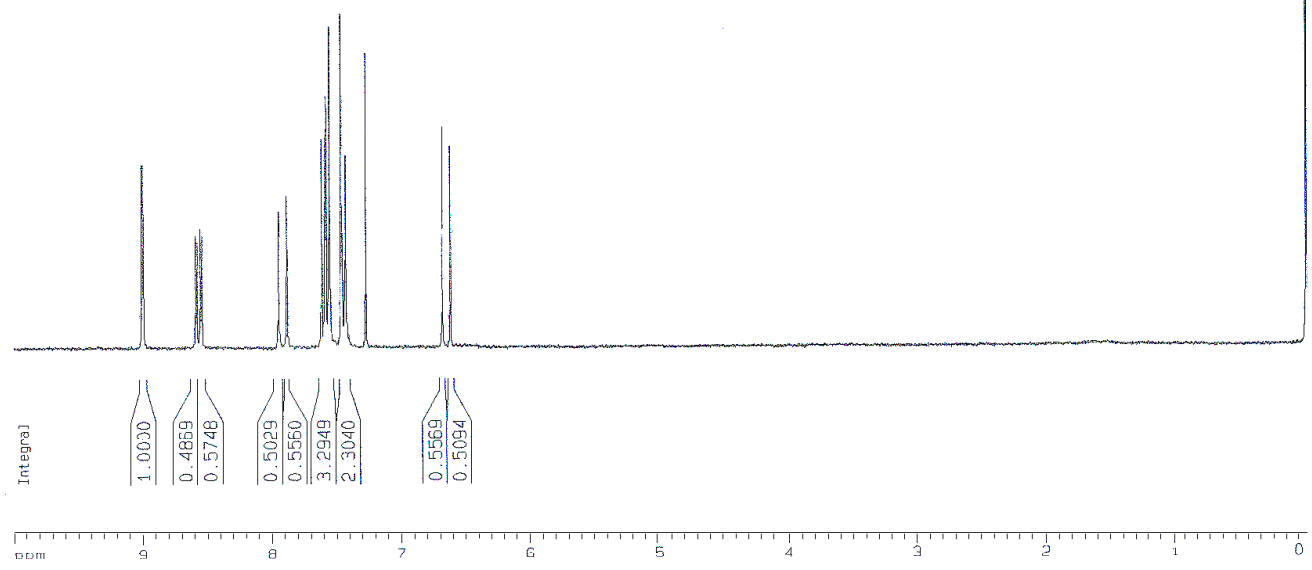


${ }^{1} \mathrm{H}$ NMR spectrum for 2,4-dinitrophenyl 4-methylcinnamate (1d)

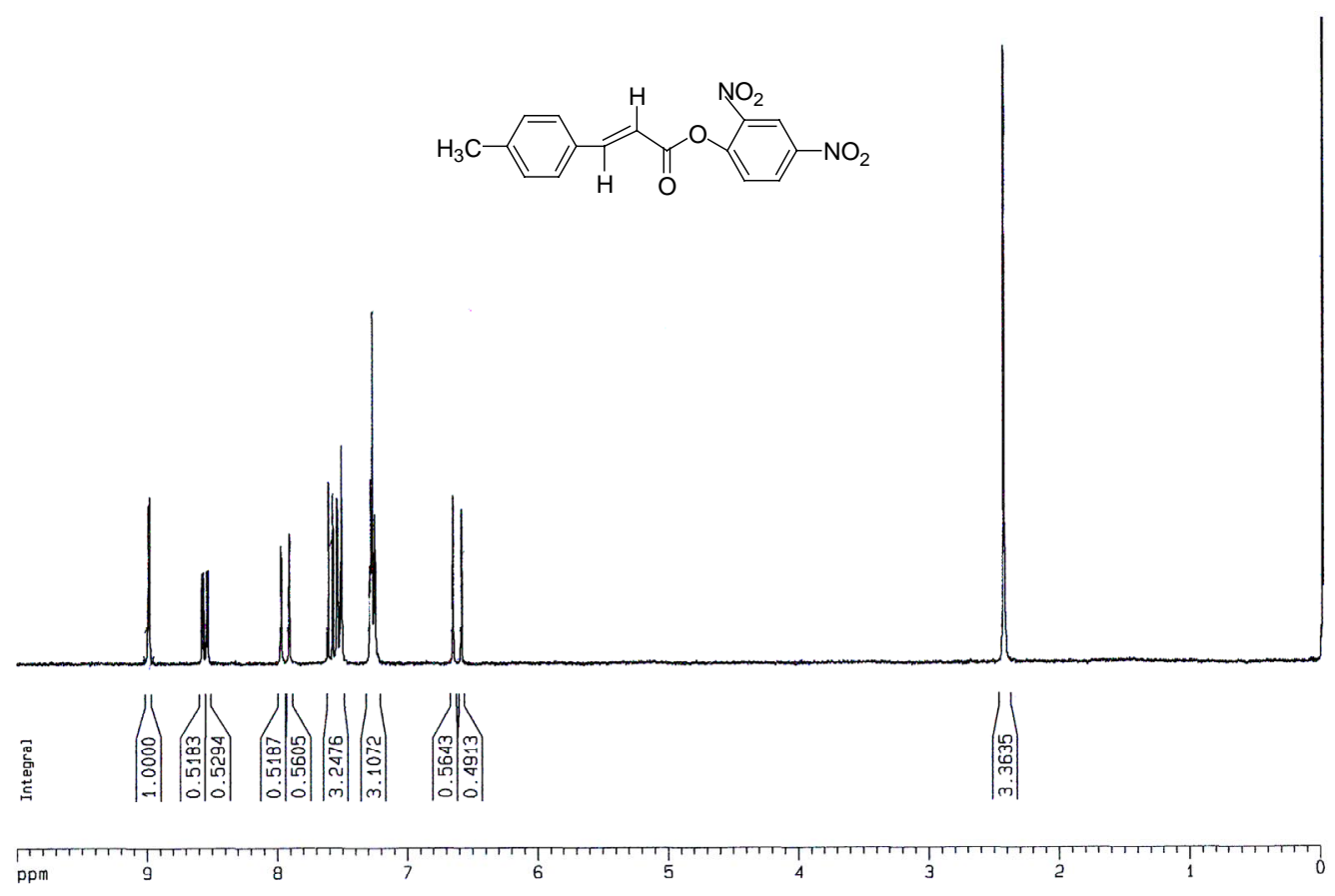

${ }^{1}$ H NMR spectrum for 2,4-dinitrophenyl 4-methoxycinnamate (1e)
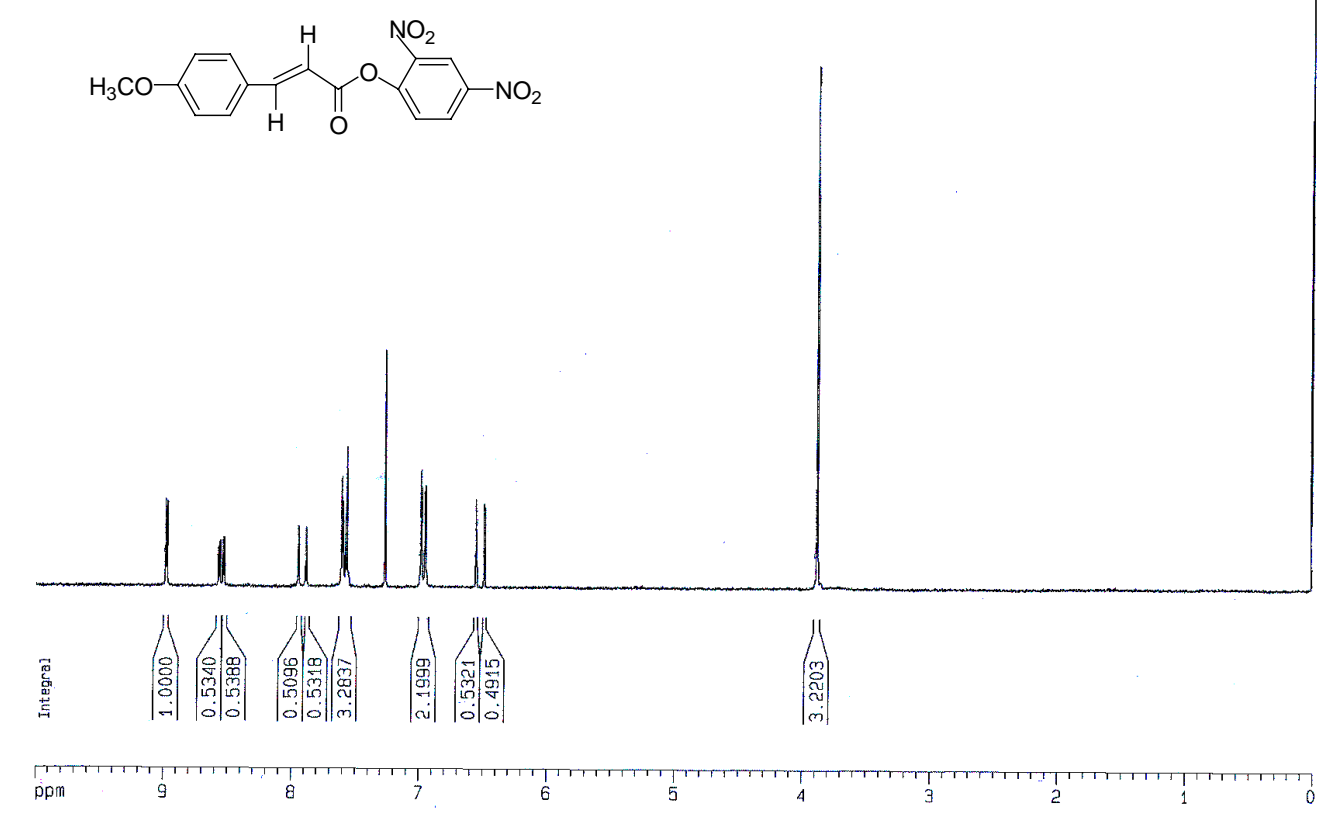
${ }^{1} \mathrm{H}$ NMR spectrum for 4-formylphenyl cinnamate (3c)

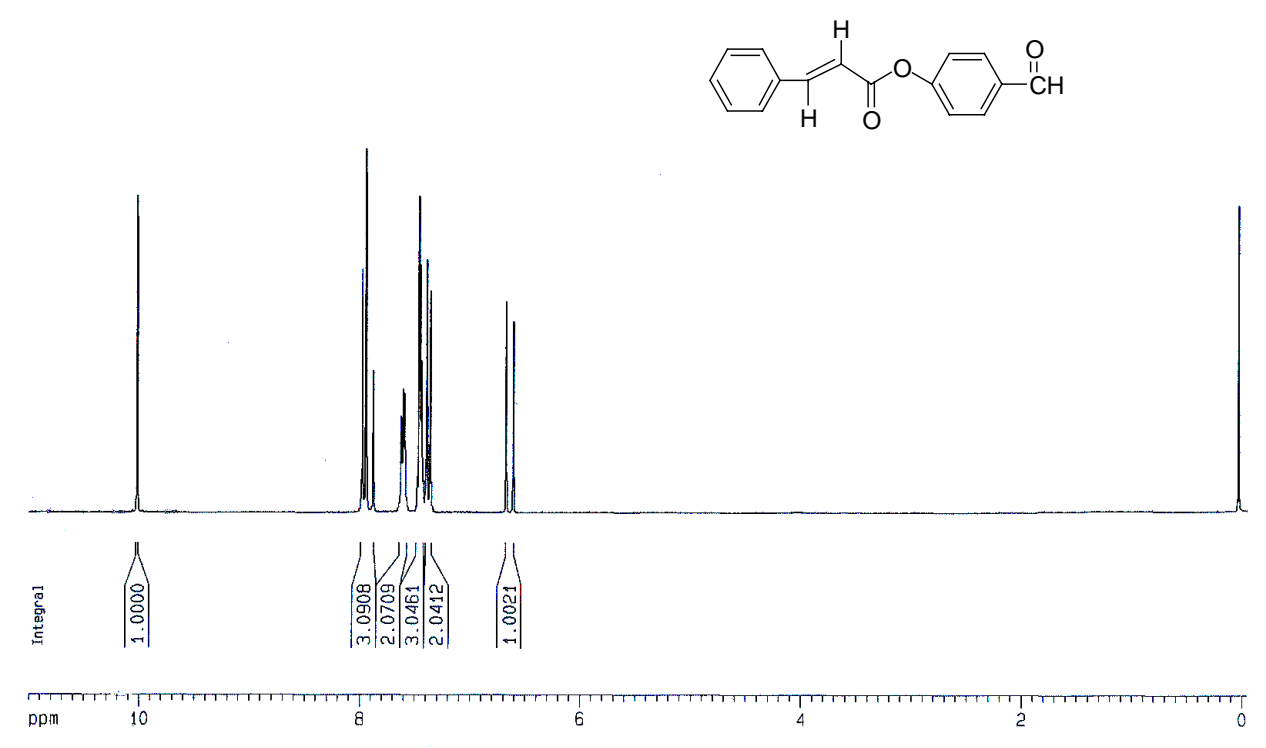

${ }^{1} \mathrm{H}$ NMR spectrum for 4-(ethoxycarbonyl)phenyl cinnamate (3e)
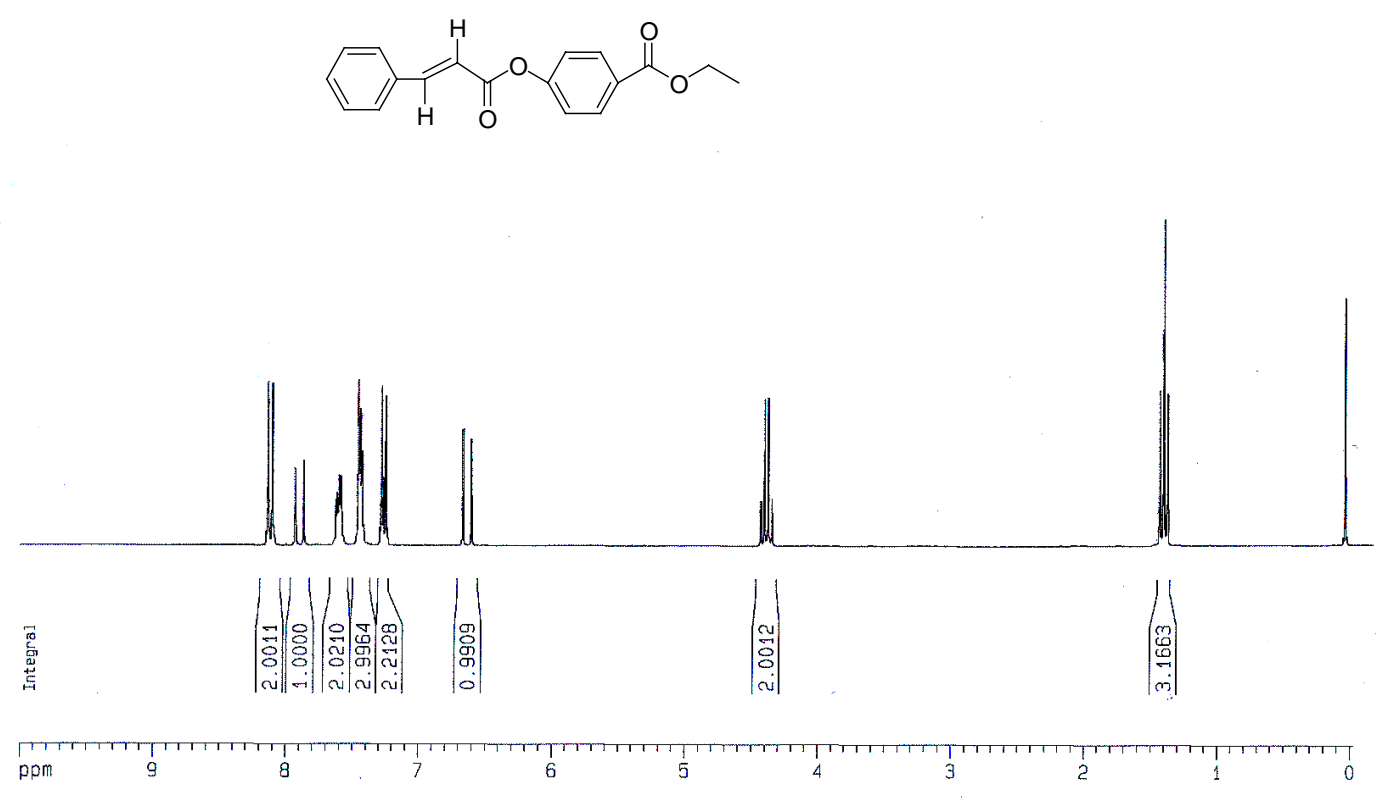
${ }^{1} \mathrm{H}$ NMR spectrum for 3-chlorophenyl cinnamate (3f)
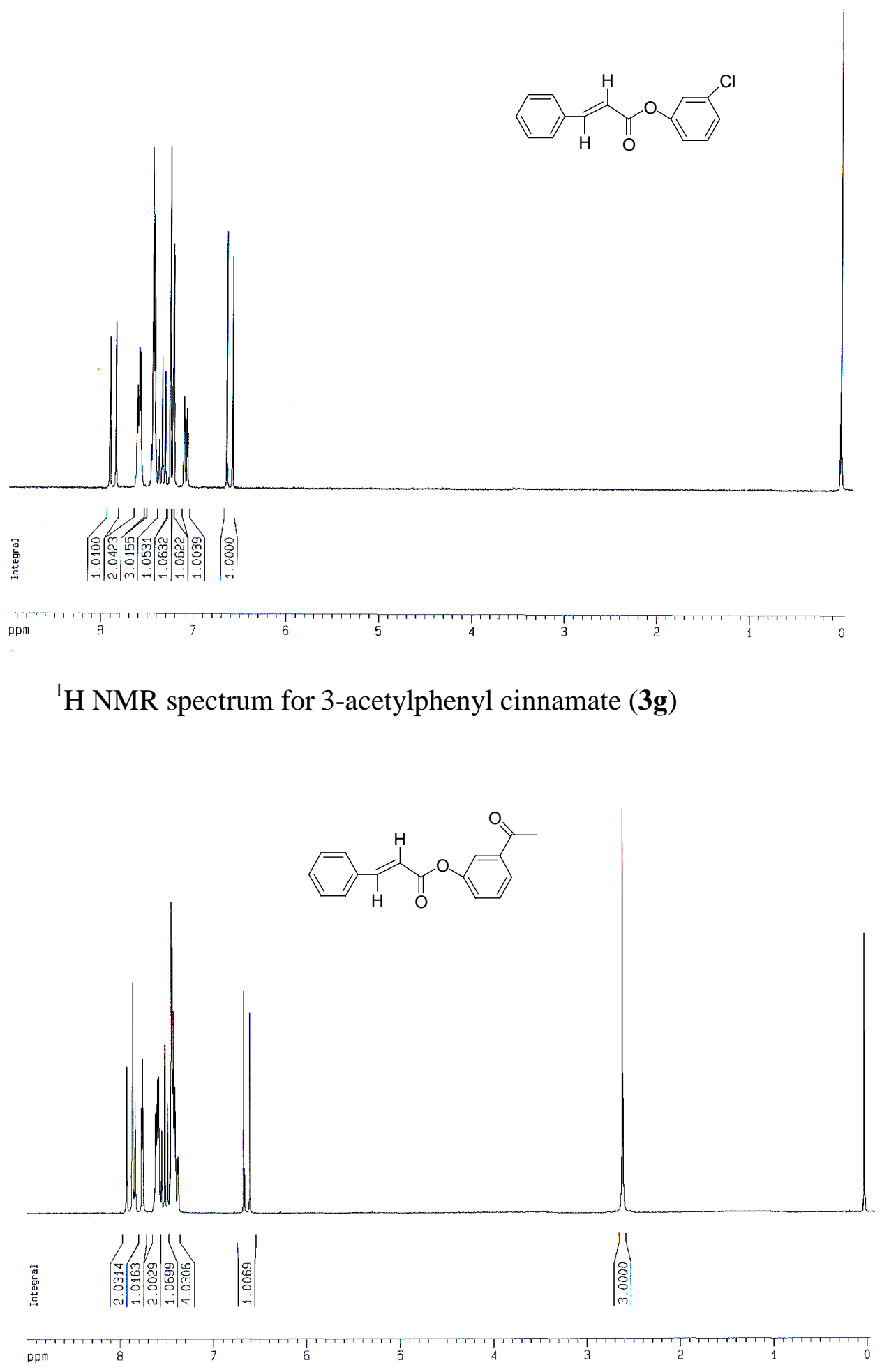
Table S1. Kinetic data for the reaction of 2,4-dinitrophenyl 4-nitrocinnamate (1a) with piperidine in $80 \mathrm{~mol} \% \mathrm{H}_{2} \mathrm{O} / 20 \mathrm{~mol} \% \mathrm{DMSO}$ at $25.0 \pm 0.1^{\circ} \mathrm{C}$.

\begin{tabular}{ccc}
\hline & {$\left[\mathrm{R}_{2} \mathrm{NH}\right] / 10^{-2} \mathrm{M}$} & $k_{\mathrm{obsd}} / \mathrm{s}^{-1}$ \\
\hline 1 & 1.01 & 6.74 \\
3 & 2.02 & 13.4 \\
4 & 3.03 & 19.4 \\
5 & 4.04 & 26.4 \\
\hline$k_{\mathrm{N}}=640 \pm 7 \mathrm{M}^{-1} \mathrm{~s}^{-1}$, & corr. coeff. $=0.9997$ & \\
\hline
\end{tabular}

Table S2. Kinetic data for the reaction of 2,4-dinitrophenyl 4-chlorocinnamate (1b) with piperidine in $80 \mathrm{~mol} \% \mathrm{H}_{2} \mathrm{O} / 20 \mathrm{~mol} \% \mathrm{DMSO}$ at $25.0 \pm 0.1^{\circ} \mathrm{C}$.

\begin{tabular}{ccc}
\hline & {$\left[\mathrm{R}_{2} \mathrm{NH}\right] / 10^{-2} \mathrm{M}$} & $k_{\mathrm{obsd}} / \mathrm{s}^{-1}$ \\
\hline 1 & 2.01 & 5.10 \\
3 & 4.02 & 10.1 \\
4 & 6.03 & 15.3 \\
5 & 8.04 & 20.3 \\
\hline$k_{\mathrm{N}}=253 \pm 1 \mathrm{M}^{-1} \mathrm{~s}^{-1}$, & corr. coeff. $=0.9999$ & \\
\hline
\end{tabular}


Table S3. Kinetic data for the reaction of 2,4-dinitrophenyl cinnamate (1c) with piperidine in $80 \mathrm{~mol} \% \mathrm{H}_{2} \mathrm{O} / 20 \mathrm{~mol} \%$ DMSO at $25.0 \pm 0.1^{\circ} \mathrm{C}$.

\begin{tabular}{ccc}
\hline & {$\left[\mathrm{R}_{2} \mathrm{NH}\right] / 10^{-3} \mathrm{M}$} & $k_{\mathrm{obsd}} / \mathrm{s}^{-1}$ \\
\hline 1 & 1.96 & 0.350 \\
2 & 3.92 & 0.770 \\
3 & 5.88 & 1.14 \\
4 & 7.84 & 1.47 \\
5 & 9.80 & 1.89 \\
\hline$k_{\mathrm{N}}=193 \pm 4 \mathrm{M}^{-1} \mathrm{~s}^{-1}, \quad$ corr. coeff. $=0.9992$ &
\end{tabular}

Table S4. Kinetic data for the reaction of 2,4-dinitrophenyl 4-methylcinnamate (1d) with piperidine in $80 \mathrm{~mol} \% \mathrm{H}_{2} \mathrm{O} / 20 \mathrm{~mol} \% \mathrm{DMSO}$ at $25.0 \pm 0.1^{\circ} \mathrm{C}$.

\begin{tabular}{ccc}
\hline & {$\left[\mathrm{R}_{2} \mathrm{NH}\right] / 10^{-2} \mathrm{M}$} & $k_{\mathrm{obsd}} / \mathrm{s}^{-1}$ \\
\hline 1 & 2.02 & 2.69 \\
2 & 4.04 & 5.50 \\
3 & 6.06 & 8.37 \\
4 & 8.08 & 11.2 \\
5 & 10.1 & 14.0 \\
\hline$k_{\mathrm{N}}=140 \pm 1 \mathrm{M}^{-1} \mathrm{~s}^{-1}, \quad$ corr. coeff. $=0.9999$ &
\end{tabular}


Table S5. Kinetic data for the reaction of 2,4-dinitrophenyl 4-methoxycinnamate (1e) with piperidine in $80 \mathrm{~mol} \% \mathrm{H}_{2} \mathrm{O} / 20 \mathrm{~mol} \% \mathrm{DMSO}$ at $25.0 \pm 0.1^{\circ} \mathrm{C}$.

\begin{tabular}{ccc}
\hline & {$\left[\mathrm{R}_{2} \mathrm{NH}\right] / 10^{-2} \mathrm{M}$} & $k_{\mathrm{obsd}} / \mathrm{s}^{-1}$ \\
\hline 1 & 2.02 & 1.85 \\
2 & 4.04 & 3.70 \\
4 & 6.06 & 5.52 \\
4 & 8.08 & 7.48 \\
5 & 10.1 & 9.31 \\
\hline$k_{\mathrm{N}}=92.5 \pm 0.6 \mathrm{M}^{-1} \mathrm{~s}^{-1}, \quad$ corr. coeff. $=0.9999$ &
\end{tabular}

Table S6. Kinetic data for the reaction of 2,4-dinitrophenyl 4-nitrocinnamate (1a) with 3methylpiperidine in $80 \mathrm{~mol} \% \mathrm{H}_{2} \mathrm{O} / 20 \mathrm{~mol} \% \mathrm{DMSO}$ at $25.0 \pm 0.1^{\circ} \mathrm{C}$.

\begin{tabular}{lcc}
\hline & {$\left[\mathrm{R}_{2} \mathrm{NH}\right] / 10^{-3} \mathrm{M}$} & $k_{\mathrm{obsd}} / \mathrm{s}^{-1}$ \\
\hline 1 & 5.55 & 3.39 \\
2 & 11.1 & 6.77 \\
3 & 16.7 & 10.0 \\
4 & 22.2 & 13.7 \\
5 & 27.8 & 17.2 \\
\hline$k_{\mathrm{N}}=621 \pm 9 \mathrm{M}^{-1} \mathrm{~s}^{-1}, \quad$ corr. coeff. $=0.9997$ &
\end{tabular}


Table S7. Kinetic data for the reaction of 2,4-dinitrophenyl cinnamate (1c) with 3methylpiperidine in $80 \mathrm{~mol} \% \mathrm{H}_{2} \mathrm{O} / 20 \mathrm{~mol} \%$ DMSO at $25.0 \pm 0.1^{\circ} \mathrm{C}$.

\begin{tabular}{ccc}
\hline & {$\left[\mathrm{R}_{2} \mathrm{NH}\right] / 10^{-2} \mathrm{M}$} & $k_{\mathrm{obsd}} / \mathrm{s}^{-1}$ \\
\hline 1 & 0.98 & 1.81 \\
2 & 1.96 & 3.58 \\
3 & 2.94 & 5.53 \\
4 & 3.92 & 7.14 \\
5 & 4.90 & 9.12 \\
\hline$k_{\mathrm{N}}=186 \pm 3 \mathrm{M}^{-1} \mathrm{~s}^{-1}, \quad$ corr. coeff. $=0.9996$ &
\end{tabular}

Table S8. Kinetic data for the reaction of 2,4-dinitrophenyl 4-methoxycinnamate (1e) with 3-methylpiperidine in $80 \mathrm{~mol} \% \mathrm{H}_{2} \mathrm{O} / 20 \mathrm{~mol} \% \mathrm{DMSO}$ at $25.0 \pm 0.1^{\circ} \mathrm{C}$.

\begin{tabular}{ccc}
\hline & {$\left[\mathrm{R}_{2} \mathrm{NH}\right] / 10^{-2} \mathrm{M}$} & $k_{\mathrm{obsd}} / \mathrm{s}^{-1}$ \\
\hline 1 & 2.02 & 1.59 \\
2 & 4.04 & 3.38 \\
3 & 6.06 & 5.15 \\
4 & 8.08 & 6.68 \\
5 & 10.1 & 8.40 \\
\hline$k_{\mathrm{N}}=83.8 \pm 1.3 \mathrm{M}^{-1} \mathrm{~s}^{-1}, \quad$ corr. coeff. $=0.9996$ &
\end{tabular}


Table S9. Kinetic data for the reaction of 2,4-dinitrophenyl 4-nitrocinnamate (1a) with piperazine in $80 \mathrm{~mol} \% \mathrm{H}_{2} \mathrm{O} / 20 \mathrm{~mol} \% \mathrm{DMSO}$ at $25.0 \pm 0.1^{\circ} \mathrm{C}$.

\begin{tabular}{ccc}
\hline & {$\left[\mathrm{R}_{2} \mathrm{NH}\right] / 10^{-3} \mathrm{M}$} & $k_{\mathrm{obsd}} / \mathrm{s}^{-1}$ \\
\hline 1 & 5.55 & 1.71 \\
3 & 11.1 & 3.18 \\
4 & 16.7 & 4.76 \\
5 & 22.2 & 6.15 \\
\hline$k_{\mathrm{N}}=264 \pm 4 \mathrm{M}^{-1} \mathrm{~s}^{-1}, \quad$ corr. coeff. $=0.9997$ &
\end{tabular}

Table S10. Kinetic data for the reaction of 2,4-dinitrophenyl cinnamate (1c) with piperazine in $80 \mathrm{~mol} \% \mathrm{H}_{2} \mathrm{O} / 20 \mathrm{~mol} \% \mathrm{DMSO}$ at $25.0 \pm 0.1^{\circ} \mathrm{C}$.

\begin{tabular}{ccc}
\hline & {$\left[\mathrm{R}_{2} \mathrm{NH}\right] / 10^{-2} \mathrm{M}$} & $k_{\mathrm{obsd}} / \mathrm{s}^{-1}$ \\
\hline 1 & 1.02 & 1.16 \\
2 & 2.04 & 2.39 \\
3 & 3.06 & 3.55 \\
4 & 4.08 & 4.71 \\
5 & 5.10 & 5.86 \\
\hline$k_{\mathrm{N}}=115 \pm 1 \mathrm{M}^{-1} \mathrm{~s}^{-1}, \quad$ corr. coeff. $=0.9999$ &
\end{tabular}


Table S11. Kinetic data for the reaction of 2,4-dinitrophenyl 4-methoxycinnamate (1e) with piperazine in $80 \mathrm{~mol} \% \mathrm{H}_{2} \mathrm{O} / 20 \mathrm{~mol} \% \mathrm{DMSO}$ at $25.0 \pm 0.1^{\circ} \mathrm{C}$.

\begin{tabular}{ccc}
\hline & {$\left[\mathrm{R}_{2} \mathrm{NH}\right] / 10^{-2} \mathrm{M}$} & $k_{\mathrm{obsd}} / \mathrm{s}^{-1}$ \\
\hline 1 & 0.995 & 0.547 \\
2 & 1.99 & 1.05 \\
3 & 2.99 & 1.55 \\
4 & 3.98 & 2.07 \\
5 & 4.98 & 2.56 \\
\hline$k_{\mathrm{N}}=50.7 \pm 0.3 \mathrm{M}^{-1} \mathrm{~s}^{-1}, \quad$ corr. coeff. $=0.9999$ &
\end{tabular}

Table S12. Kinetic data for the reaction of 2,4-dinitrophenyl 4-nitrocinnamate (1a) with morpholine in $80 \mathrm{~mol} \% \mathrm{H}_{2} \mathrm{O} / 20 \mathrm{~mol} \% \mathrm{DMSO}$ at $25.0 \pm 0.1^{\circ} \mathrm{C}$.

\begin{tabular}{ccc}
\hline & {$\left[\mathrm{R}_{2} \mathrm{NH}\right] / 10^{-3} \mathrm{M}$} & $k_{\mathrm{obsd}} / 10^{-1} \mathrm{~s}^{-1}$ \\
\hline 1 & 0.790 & 0.483 \\
3 & 1.58 & 0.948 \\
4 & 2.36 & 1.49 \\
5 & 3.13 & 1.94 \\
\hline$k_{\mathrm{N}}=65.0 \pm 2 \mathrm{M}^{-1} \mathrm{~s}^{-1}, \quad$ corr. coeff. $=0.9991$ & \\
\hline
\end{tabular}


Table S13. Kinetic data for the reaction of 2,4-dinitrophenyl 4-chlorocinnamate (1b) with morpholine in $80 \mathrm{~mol} \% \mathrm{H}_{2} \mathrm{O} / 20 \mathrm{~mol} \% \mathrm{DMSO}$ at $25.0 \pm 0.1^{\circ} \mathrm{C}$.

\begin{tabular}{ccc}
\hline & {$\left[\mathrm{R}_{2} \mathrm{NH}\right] / 10^{-3} \mathrm{M}$} & $k_{\mathrm{obsd}} / 10^{-2} \mathrm{~s}^{-1}$ \\
\hline 1 & 0.800 & 1.96 \\
3 & 1.58 & 4.27 \\
4 & 2.37 & 6.44 \\
5 & 3.14 & 8.42 \\
\hline$k_{\mathrm{N}}=27.0 \pm 0.05 \mathrm{M}^{-1} \mathrm{~s}^{-1}, \quad$ corr. coeff. $=0.9995$ & 10.4
\end{tabular}

Table S14. Kinetic data for the reaction of 2,4-dinitrophenyl cinnamate (1c) with morpholine in $80 \mathrm{~mol} \% \mathrm{H}_{2} \mathrm{O} / 20 \mathrm{~mol} \% \mathrm{DMSO}$ at $25.0 \pm 0.1^{\circ} \mathrm{C}$.

\begin{tabular}{ccc}
\hline & {$\left[\mathrm{R}_{2} \mathrm{NH}\right] / 10^{-3} \mathrm{M}$} & $k_{\text {obsd }} / 10^{-2} \mathrm{~s}^{-1}$ \\
\hline 1 & 0.79 & 1.64 \\
3 & 1.58 & 3.46 \\
4 & 2.36 & 5.21 \\
5 & 3.13 & 6.98 \\
\hline$k_{\mathrm{N}}=22.7 \pm 0.05 \mathrm{M}^{-1} \mathrm{~s}^{-1}, \quad$ corr. coeff. $=0.9999$ &
\end{tabular}


Table S15. Kinetic data for the reaction of 2,4-dinitrophenyl 4-methylcinnamate (1d) with morpholine in $80 \mathrm{~mol} \% \mathrm{H}_{2} \mathrm{O} / 20 \mathrm{~mol} \% \mathrm{DMSO}$ at $25.0 \pm 0.1^{\circ} \mathrm{C}$.

\begin{tabular}{ccc}
\hline & {$\left[\mathrm{R}_{2} \mathrm{NH}\right] / 10^{-2} \mathrm{M}$} & $k_{\mathrm{obsd}} / 10^{-2} \mathrm{~s}^{-1}$ \\
\hline 1 & 0.800 & 1.20 \\
3 & 1.58 & 2.42 \\
4 & 2.37 & 3.68 \\
5 & 3.14 & 4.79 \\
\hline$k_{\mathrm{N}}=15.8 \pm 0.3 \mathrm{M}^{-1} \mathrm{~s}^{-1}, \quad$ corr. coeff. $=0.9995$ &
\end{tabular}

Table S16. Kinetic data for the reaction of 2,4-dinitrophenyl 4-methoxycinnamate (1e) with morpholine in $80 \mathrm{~mol} \% \mathrm{H}_{2} \mathrm{O} / 20 \mathrm{~mol} \% \mathrm{DMSO}$ at $25.0 \pm 0.1^{\circ} \mathrm{C}$.

\begin{tabular}{ccc}
\hline & {$\left[\mathrm{R}_{2} \mathrm{NH}\right] / 10^{-3} \mathrm{M}$} & $k_{\mathrm{obsd}} / 10^{-2} \mathrm{~s}^{-1}$ \\
\hline 1 & 1.58 & 1.63 \\
3 & 3.14 & 3.33 \\
4 & 4.68 & 4.85 \\
5 & 6.19 & 6.46 \\
\hline$k_{\mathrm{N}}=10.6 \pm 0.1 \mathrm{M}^{-1} \mathrm{~s}^{-1}, \quad$ corr. coeff. $=0.9997$ & \\
\hline
\end{tabular}


Table S17. Kinetic data for the reaction of 2,4-dinitrophenyl 4-nitrocinnamate (1a) with 1formylpiperazine in $80 \mathrm{~mol} \% \mathrm{H}_{2} \mathrm{O} / 20 \mathrm{~mol} \% \mathrm{DMSO}$ at $25.0 \pm 0.1^{\circ} \mathrm{C}$.

\begin{tabular}{ccc}
\hline & {$\left[\mathrm{R}_{2} \mathrm{NH}\right] / 10^{-3} \mathrm{M}$} & $k_{\mathrm{obsd}} / 10^{-2} \mathrm{~s}^{-1}$ \\
\hline 1 & 1.65 & 3.24 \\
3 & 3.28 & 6.53 \\
4 & 4.88 & 9.56 \\
5 & 6.45 & 13.3 \\
\hline & 8.00 & 16.3 \\
\hline$k_{\mathrm{N}}=20.7 \pm 0.4 \mathrm{M}^{-1} \mathrm{~s}^{-1}, \quad$ corr. coeff. $=0.9994$ &
\end{tabular}

Table S18. Kinetic data for the reaction of 2,4-dinitrophenyl cinnamate (1c) with 1formylpiperazine in $80 \mathrm{~mol} \mathrm{\%} \mathrm{H}_{2} \mathrm{O} / 20 \mathrm{~mol} \% \mathrm{DMSO}$ at $25.0 \pm 0.1^{\circ} \mathrm{C}$.

\begin{tabular}{ccc}
\hline & {$\left[\mathrm{R}_{2} \mathrm{NH}\right] / 10^{-3} \mathrm{M}$} & $k_{\mathrm{obsd}} / 10^{-2} \mathrm{~s}^{-1}$ \\
\hline 1 & 1.62 & 1.20 \\
3 & 3.22 & 2.31 \\
4 & 4.78 & 3.57 \\
5 & 6.33 & 4.66 \\
& 7.85 & 5.76
\end{tabular}

$k_{\mathrm{N}}=7.37 \pm 0.10 \mathrm{M}^{-1} \mathrm{~s}^{-1}, \quad$ corr. coeff. $=0.9997$ 
Table S19. Kinetic data for the reaction of 2,4-dinitrophenyl 4-methoxycinnamate (1e) with 1-formylpiperazine in $80 \mathrm{~mol} \% \mathrm{H}_{2} \mathrm{O} / 20 \mathrm{~mol} \%$ DMSO at $25.0 \pm 0.1^{\circ} \mathrm{C}$.

\begin{tabular}{ccc}
\hline & {$\left[\mathrm{R}_{2} \mathrm{NH}\right] / 10^{-2} \mathrm{M}$} & $k_{\text {obsd }} / 10^{-2} \mathrm{~s}^{-1}$ \\
\hline 1 & 0.400 & 1.42 \\
2 & 0.785 & 2.79 \\
3 & 1.15 & 4.15 \\
4 & 1.51 & 5.30 \\
5 & 1.85 & 6.43 \\
\hline$k_{\mathrm{N}}=3.46 \pm 0.06 \mathrm{M}^{-1} \mathrm{~s}^{-1}, \quad$ corr. coeff. $=0.9995$ &
\end{tabular}

Table S20. Kinetic data for the reaction of 2,4-dinitrophenyl 4-nitrocinnamate (1a) with piperazinium ion in $80 \mathrm{~mol} \% \mathrm{H}_{2} \mathrm{O} / 20 \mathrm{~mol} \%$ DMSO at $25.0 \pm 0.1^{\circ} \mathrm{C}$.

\begin{tabular}{ccc}
\hline & {$\left[\mathrm{R}_{2} \mathrm{NH}\right] / 10^{-2} \mathrm{M}$} & $k_{\mathrm{obsd}} / 10^{-2} \mathrm{~s}^{-1}$ \\
\hline 1 & 0.926 & 1.36 \\
2 & 1.77 & 2.55 \\
3 & 2.54 & 3.65 \\
4 & 3.24 & 4.74 \\
5 & 3.89 & 5.73 \\
$k_{\mathrm{N}}=1.48 \pm 0.02 \mathrm{M}^{-1} \mathrm{~s}^{-1}, \quad$ corr. coeff. $=0.9997$ &
\end{tabular}


Table S21. Kinetic data for the reaction of 2,4-dinitrophenyl 4-chlorocinnamate (1b) with piperazinium ion in $80 \mathrm{~mol} \% \mathrm{H}_{2} \mathrm{O} / 20 \mathrm{~mol} \% \mathrm{DMSO}$ at $25.0 \pm 0.1^{\circ} \mathrm{C}$.

\begin{tabular}{ccc}
\hline & {$\left[\mathrm{R}_{2} \mathrm{NH}\right] / 10^{-2} \mathrm{M}$} & $k_{\mathrm{obsd}} / 10^{-2} \mathrm{~s}^{-1}$ \\
\hline 1 & 0.926 & 0.686 \\
3 & 1.77 & 1.27 \\
4 & 2.54 & 1.91 \\
5 & 3.24 & 2.43 \\
\hline$k_{\mathrm{N}}=0.748 \pm 0.010 \mathrm{M}^{-1} \mathrm{~s}^{-1}, \quad$ corr. coeff. $=0.9995$ & 2.87
\end{tabular}

Table S22. Kinetic data for the reaction of 2,4-dinitrophenyl cinnamate (1c) with piperazinium ion in $80 \mathrm{~mol} \% \mathrm{H}_{2} \mathrm{O} / 20 \mathrm{~mol} \% \mathrm{DMSO}$ at $25.0 \pm 0.1^{\circ} \mathrm{C}$.

\begin{tabular}{ccc}
\hline & {$\left[\mathrm{R}_{2} \mathrm{NH}\right] / 10^{-2} \mathrm{M}$} & $k_{\text {obsd }} / 10^{-2} \mathrm{~s}^{-1}$ \\
\hline 1 & 0.752 & 0.530 \\
3 & 1.45 & 1.02 \\
4 & 2.10 & 1.48 \\
5 & 2.70 & 1.91 \\
\hline$k_{\mathrm{N}}=0.707 \pm 0.001 \mathrm{M}^{-1} \mathrm{~s}^{-1}, \quad$ corr. coeff. $=0.9999$ & 2.30
\end{tabular}


Table S23. Kinetic data for the reaction of 2,4-dinitrophenyl 4-methylcinnamate (1d) with piperazinium ion in $80 \mathrm{~mol} \% \mathrm{H}_{2} \mathrm{O} / 20 \mathrm{~mol} \% \mathrm{DMSO}$ at $25.0 \pm 0.1^{\circ} \mathrm{C}$.

\begin{tabular}{ccc}
\hline & {$\left[\mathrm{R}_{2} \mathrm{NH}\right] / 10^{-2} \mathrm{M}$} & $k_{\mathrm{obsd}} / 10^{-3} \mathrm{~s}^{-1}$ \\
\hline 1 & 0.931 & 4.66 \\
2 & 1.77 & 8.99 \\
3 & 2.55 & 12.9 \\
4 & 3.26 & 16.4 \\
5 & 3.91 & 19.6 \\
\hline$k_{\mathrm{N}}=0.501 \pm 0.003 \mathrm{M}^{-1} \mathrm{~s}^{-1}, \quad$ corr. coeff. $=0.9999$ &
\end{tabular}

Table S24. Kinetic data for the reaction of 2,4-dinitrophenyl 4-methoxycinnamate (1e) with piperazinium ion in $80 \mathrm{~mol} \% \mathrm{H}_{2} \mathrm{O} / 20 \mathrm{~mol} \% \mathrm{DMSO}$ at $25.0 \pm 0.1^{\circ} \mathrm{C}$.

\begin{tabular}{ccc}
\hline & {$\left[\mathrm{R}_{2} \mathrm{NH}\right] / 10^{-2} \mathrm{M}$} & $k_{\mathrm{obsd}} / 10^{-3} \mathrm{~s}^{-1}$ \\
\hline 1 & 0.931 & 3.32 \\
3 & 1.77 & 6.20 \\
4 & 2.55 & 8.93 \\
5 & 3.26 & 11.6 \\
\hline$k_{\mathrm{N}}=0.354 \pm 0.003 \mathrm{M}^{-1} \mathrm{~s}^{-1}, \quad$ corr. coeff. $=0.9998$ & 13.8
\end{tabular}


Table S25. Kinetic data for the reaction of 3,4-dinitrophenyl cinnamate (3a) with piperidine in $80 \mathrm{~mol} \% \mathrm{H}_{2} \mathrm{O} / 20 \mathrm{~mol} \% \mathrm{DMSO}$ at $25.0 \pm 0.1^{\circ} \mathrm{C}$.

\begin{tabular}{ccc}
\hline & {$\left[\mathrm{R}_{2} \mathrm{NH}\right] / 10^{-2} \mathrm{M}$} & $k_{\mathrm{obsd}} / \mathrm{s}^{-1}$ \\
\hline 1 & 1.01 & 1.40 \\
2 & 2.02 & 2.95 \\
3 & 3.03 & 4.55 \\
4 & 4.04 & 6.10 \\
5 & 5.05 & 7.69 \\
$k_{\mathrm{N}}=156 \pm 1 \mathrm{M}^{-1} \mathrm{~s}^{-1}, \quad$ corr. coeff. $=0.9999$ &
\end{tabular}

Table S26. Kinetic data for the reaction of 4-nitrophenyl cinnamate (3b) with piperidine in $80 \mathrm{~mol} \% \mathrm{H}_{2} \mathrm{O} / 20 \mathrm{~mol} \%$ DMSO at $25.0 \pm 0.1^{\circ} \mathrm{C}$.

\begin{tabular}{ccc}
\hline & {$\left[\mathrm{R}_{2} \mathrm{NH}\right] / 10^{-3} \mathrm{M}$} & $k_{\mathrm{obsd}} / 10^{-2} \mathrm{~s}^{-1}$ \\
\hline 1 & 0.803 & 0.961 \\
2 & 1.60 & 1.99 \\
3 & 2.39 & 2.97 \\
4 & 3.17 & 3.88 \\
5 & 3.95 & 4.87 \\
\hline
\end{tabular}

$k_{\mathrm{N}}=12.3 \pm 0.1 \mathrm{M}^{-1} \mathrm{~s}^{-1}, \quad$ corr. coeff. $=0.9998$ 
Table S27. Kinetic data for the reaction of 4-formylphenyl cinnamate (3c) with piperidine in $80 \mathrm{~mol} \% \mathrm{H}_{2} \mathrm{O} / 20 \mathrm{~mol} \%$ DMSO at $25.0 \pm 0.1^{\circ} \mathrm{C}$.

\begin{tabular}{ccc}
\hline & {$\left[\mathrm{R}_{2} \mathrm{NH}\right] / 10^{-3} \mathrm{M}$} & $k_{\text {obsd }} / 10^{-2} \mathrm{~s}^{-1}$ \\
\hline 1 & 1.72 & 0.743 \\
3 & 3.41 & 1.39 \\
4 & 5.08 & 2.07 \\
5 & 6.72 & 2.79 \\
\hline$k_{\mathrm{N}}=4.03 \pm 0.06 \mathrm{M}^{-1} \mathrm{~s}^{-1}, \quad$ corr. coeff. $=0.9996$ & 3.37 \\
\hline
\end{tabular}

Table S28. Kinetic data for the reaction of 4-acetylphenyl cinnamate (3d) with piperidine in $80 \mathrm{~mol} \% \mathrm{H}_{2} \mathrm{O} / 20 \mathrm{~mol} \% \mathrm{DMSO}$ at $25.0 \pm 0.1^{\circ} \mathrm{C}$.

\begin{tabular}{ccc}
\hline & {$\left[\mathrm{R}_{2} \mathrm{NH}\right] / 10^{-2} \mathrm{M}$} & $\mathrm{k}_{\mathrm{obsd}} / 10^{-2} \mathrm{~s}^{-1}$ \\
\hline 1 & 0.392 & 0.702 \\
3 & 0.769 & 1.34 \\
4 & 1.13 & 1.93 \\
5 & 1.48 & 2.56 \\
& 1.82 & 3.14
\end{tabular}

$\mathrm{k}_{\mathrm{N}}=1.71 \pm 0.01 \mathrm{M}^{-1} \mathrm{~s}^{-1}, \quad$ corr. coeff. $=0.9999$ 
Table 29. Kinetic data for the reaction of 4-(ethoxycarbonyl)phenyl Cinnamate (3e) with piperidine in $80 \mathrm{~mol} \% \mathrm{H}_{2} \mathrm{O} / 20 \mathrm{~mol} \% \mathrm{DMSO}$ at $25.0 \pm 0.1^{\circ} \mathrm{C}$.

\begin{tabular}{ccc}
\hline & {$\left[\mathrm{R}_{2} \mathrm{NH}\right] / 10^{-2} \mathrm{M}$} & $\mathrm{k}_{\mathrm{obsd}} / 10^{-2} \mathrm{~s}^{-1}$ \\
\hline 1 & 0.788 & 0.999 \\
3 & 1.52 & 1.89 \\
4 & 2.20 & 2.71 \\
5 & 2.83 & 3.57 \\
\hline $\mathrm{k}_{\mathrm{N}}=1.24 \pm 0.02 \mathrm{M}^{-1} \mathrm{~s}^{-1}, \quad$ corr. coeff. $=0.9997$ & \\
\hline
\end{tabular}

Table S30. Kinetic data for the reaction of 3-chlorophenyl cinnamate (3f) with piperidine in $80 \mathrm{~mol} \% \mathrm{H}_{2} \mathrm{O} / 20 \mathrm{~mol} \% \mathrm{DMSO}$ at $25.0 \pm 0.1^{\circ} \mathrm{C}$.

\begin{tabular}{ccc}
\hline & {$\left[\mathrm{R}_{2} \mathrm{NH}\right] / 10^{-2} \mathrm{M}$} & $\mathrm{k}_{\mathrm{obsd}} / 10^{-2} \mathrm{~s}^{-1}$ \\
\hline 1 & 1.85 & 0.572 \\
2 & 3.38 & 1.03 \\
3 & 4.68 & 1.41 \\
4 & 5.80 & 1.75 \\
5 & 6.77 & 2.03
\end{tabular}

$\mathrm{k}_{\mathrm{N}}=0.297 \pm 0.001 \mathrm{M}^{-1} \mathrm{~s}^{-1}, \quad$ corr. coeff. $=0.9999$ 
Table S31. Kinetic data for the reaction of 3-acetylphenyl cinnamate (3g) with piperidine in $80 \mathrm{~mol} \% \mathrm{H}_{2} \mathrm{O} / 20 \mathrm{~mol} \%$ DMSO at $25.0 \pm 0.1^{\circ} \mathrm{C}$.

\begin{tabular}{ccc}
\hline & {$\left[\mathrm{R}_{2} \mathrm{NH}\right] / 10^{-2} \mathrm{M}$} & $\mathrm{k}_{\mathrm{obsd}} / 10^{-3} \mathrm{~s}^{-1}$ \\
\hline 1 & 1.85 & 2.93 \\
3 & 3.40 & 5.47 \\
4 & 4.71 & 7.35 \\
5 & 5.83 & 9.29 \\
\hline $\mathrm{k}_{\mathrm{N}}=0.160 \pm 0.003 \mathrm{M}^{-1} \mathrm{~s}^{-1}, \quad$ corr. coeff. $=0.9996$ & 10.9
\end{tabular}

Table S32. Kinetic data for the reaction of 3,4-dinitrophenyl cinnamate (3a) with morpholine in $80 \mathrm{~mol} \% \mathrm{H}_{2} \mathrm{O} / 20 \mathrm{~mol} \% \mathrm{DMSO}$ at $25.0 \pm 0.1^{\circ} \mathrm{C}$.

\begin{tabular}{ccc}
\hline & {$\left[\mathrm{R}_{2} \mathrm{NH}\right] / 10^{-3} \mathrm{M}$} & $k_{\mathrm{obsd}} / 10^{-2} \mathrm{~s}^{-1}$ \\
\hline 1 & 1.60 & 2.15 \\
3 & 3.17 & 4.26 \\
4 & 4.73 & 6.25 \\
5 & 6.25 & 8.12 \\
\hline$k_{\mathrm{N}}=13.2 \pm 0.3 \mathrm{M}^{-1} \mathrm{~s}^{-1}, \quad$ corr. coeff. $=0.9994$ &
\end{tabular}


Table S33. Kinetic data for the reaction of 4-nitrophenyl cinnamate (3b) with morpholine in $80 \mathrm{~mol} \% \mathrm{H}_{2} \mathrm{O} / 20 \mathrm{~mol} \%$ DMSO at $25.0 \pm 0.1^{\circ} \mathrm{C}$.

\begin{tabular}{ccc}
\hline & {$\left[\mathrm{R}_{2} \mathrm{NH}\right] / 10^{-2} \mathrm{M}$} & $k_{\mathrm{obsd}} / 10^{-2} \mathrm{~s}^{-1}$ \\
\hline 1 & 0.950 & 0.583 \\
3 & 1.81 & 0.988 \\
4 & 2.63 & 1.42 \\
5 & 3.33 & 1.82 \\
\hline$k_{\mathrm{N}}=$ & $0.531 \pm 0.004 \mathrm{M}^{-1} \mathrm{~s}^{-1}, \quad$ corr. coeff. $=0.9999$
\end{tabular}

Table S34. Kinetic data for the reaction of 4-formylphenyl cinnamate (3c) with morpholine in $80 \mathrm{~mol} \% \mathrm{H}_{2} \mathrm{O} / 20 \mathrm{~mol} \%$ DMSO at $25.0 \pm 0.1^{\circ} \mathrm{C}$.

\begin{tabular}{ccc}
\hline & {$\left[\mathrm{R}_{2} \mathrm{NH}\right] / 10^{-2} \mathrm{M}$} & $k_{\mathrm{obsd}} / 10^{-3} \mathrm{~s}^{-1}$ \\
\hline 1 & 1.83 & 1.74 \\
3 & 3.36 & 3.13 \\
4 & 4.65 & 4.54 \\
5 & 5.76 & 5.52 \\
& 6.72 & 6.45
\end{tabular}

$k_{\mathrm{N}}=0.0970 \pm 0.0017 \mathrm{M}^{-1} \mathrm{~s}^{-1}, \quad$ corr. coeff. $=0.9995$ 
Table S35. Kinetic data for the reaction of 4-acetylphenyl cinnamate (3d) with morpholine in $80 \mathrm{~mol} \% \mathrm{H}_{2} \mathrm{O} / 20 \mathrm{~mol} \%$ DMSO at $25.0 \pm 0.1^{\circ} \mathrm{C}$.

\begin{tabular}{ccc}
\hline & {$\left[\mathrm{R}_{2} \mathrm{NH}\right] / 10^{-2} \mathrm{M}$} & $\mathrm{k}_{\mathrm{obsd}} / 10^{-3} \mathrm{~s}^{-1}$ \\
\hline 1 & 1.85 & 0.574 \\
2 & 3.38 & 1.03 \\
3 & 4.06 & 1.25 \\
4 & 5.26 & 1.59 \\
5 & 5.80 & 1.74 \\
\hline $\mathrm{k}_{\mathrm{N}}=0.0296 \pm 0.0004 \mathrm{M}^{-1} \mathrm{~s}^{-1}, \quad$ corr. coeff. $=0.9997$
\end{tabular}

Table 36. Kinetic data for the reaction of 4-(ethoxycarbonyl)phenyl Cinnamate (3e) with morpholine in $80 \mathrm{~mol} \% \mathrm{H}_{2} \mathrm{O} / 20 \mathrm{~mol} \% \mathrm{DMSO}$ at $25.0 \pm 0.1^{\circ} \mathrm{C}$.

\begin{tabular}{ccc}
\hline & {$\left[\mathrm{R}_{2} \mathrm{NH}\right] / 10^{-2} \mathrm{M}$} & $\mathrm{k}_{\mathrm{obsd}} / 10^{-3} \mathrm{~s}^{-1}$ \\
\hline 1 & 4.27 & 0.889 \\
3 & 5.80 & 1.23 \\
4 & 6.77 & 1.46 \\
5 & 8.12 & 1.76 \\
\hline $\mathrm{k}_{\mathrm{N}}=0.0226 \pm 0.0001 \mathrm{M}^{-1} \mathrm{~s}^{-1}, \quad$ corr. coeff. $=0.9999$
\end{tabular}


Table S37. Kinetic data for the reaction of 3-chlorophenyl cinnamate (3f) with morpholine in $80 \mathrm{~mol} \% \mathrm{H}_{2} \mathrm{O} / 20 \mathrm{~mol} \%$ DMSO at $25.0 \pm 0.1^{\circ} \mathrm{C}$.

\begin{tabular}{ccc}
\hline & {$\left[\mathrm{R}_{2} \mathrm{NH}\right] / 10^{-2} \mathrm{M}$} & $\mathrm{k}_{\mathrm{obsd}} / 10^{-4} \mathrm{~s}^{-1}$ \\
\hline 1 & 1.83 & 0.741 \\
3 & 3.35 & 1.30 \\
4 & 4.64 & 1.84 \\
5 & 5.74 & 2.28 \\
\hline $\mathrm{k}_{\mathrm{N}}=0.00394 \pm 0.00005 \mathrm{M}^{-1} \mathrm{~s}^{-1}, \quad$ corr. coeff. $=0.9997$
\end{tabular}

Table S38. Kinetic data for the reaction of 3-acetylphenyl cinnamate (3g) with morpholine in $80 \mathrm{~mol} \% \mathrm{H}_{2} \mathrm{O} / 20 \mathrm{~mol} \%$ DMSO at $25.0 \pm 0.1^{\circ} \mathrm{C}$.

\begin{tabular}{ccc}
\hline & {$\left[\mathrm{R}_{2} \mathrm{NH}\right] / 10^{-2} \mathrm{M}$} & $\mathrm{k}_{\mathrm{obsd}} / 10^{-4} \mathrm{~s}^{-1}$ \\
\hline 1 & 1.84 & 2.67 \\
2 & 3.36 & 2.92 \\
4 & 4.65 & 3.11 \\
5 & 5.76 & 3.29 \\
5 & 6.72 & 3.46 \\
\hline
\end{tabular}

$\mathrm{k}_{\mathrm{N}}=0.00160 \pm 0.00002 \mathrm{M}^{-1} \mathrm{~s}^{-1}, \quad$ corr. coeff. $=0.9996$ 
Table 39. Kinetic data for the reaction of 4-(ethoxycarbonyl)phenyl benzoate (4e) with piperidine in $80 \mathrm{~mol} \% \mathrm{H}_{2} \mathrm{O} / 20 \mathrm{~mol} \% \mathrm{DMSO}$ at $25.0 \pm 0.1^{\circ} \mathrm{C}$.

\begin{tabular}{ccc}
\hline & {$\left[\mathrm{R}_{2} \mathrm{NH}\right] / 10^{-2} \mathrm{M}$} & $\mathrm{k}_{\mathrm{obsd}} / 10^{-2} \mathrm{~s}^{-1}$ \\
\hline 1 & 2.52 & 0.356 \\
2 & 4.51 & 0.622 \\
4 & 6.11 & 0.845 \\
5 & 7.44 & 1.03 \\
5 & 8.56 & 1.20 \\
$\mathrm{k}_{\mathrm{N}}=0.139 \pm 0.002 \mathrm{M}^{-1} \mathrm{~s}^{-1}, \quad$ corr. coeff. $=0.9997$
\end{tabular}

Table S40. Kinetic data for the reaction of 3,4-dinitrophenyl benzoate (4a) with morpholine in $80 \mathrm{~mol} \% \mathrm{H}_{2} \mathrm{O} / 20 \mathrm{~mol} \%$ DMSO at $25.0 \pm 0.1^{\circ} \mathrm{C}$.

\begin{tabular}{ccc}
\hline & {$\left[\mathrm{R}_{2} \mathrm{NH}\right] / 10^{-2} \mathrm{M}$} & $k_{\mathrm{obsd}} / 10^{-1} \mathrm{~s}^{-1}$ \\
\hline 1 & 0.418 & 0.407 \\
3 & 0.819 & 0.830 \\
4 & 1.21 & 1.19 \\
5 & 1.58 & 1.56 \\
\hline
\end{tabular}

$k_{\mathrm{N}}=10.1 \pm 0.2 \mathrm{M}^{-1} \mathrm{~s}^{-1}, \quad$ corr. coeff. $=0.9995$ 
Table S41. Kinetic data for the reaction of 4-formylphenyl benzoate (4c) with morpholine in $80 \mathrm{~mol} \% \mathrm{H}_{2} \mathrm{O} / 20 \mathrm{~mol} \%$ DMSO at $25.0 \pm 0.1^{\circ} \mathrm{C}$.

\begin{tabular}{ccc}
\hline & {$\left[\mathrm{R}_{2} \mathrm{NH}\right] / 10^{-2} \mathrm{M}$} & $k_{\mathrm{obsd}} / 10^{-3} \mathrm{~s}^{-1}$ \\
\hline 1 & 4.29 & 4.35 \\
3 & 5.83 & 5.69 \\
4 & 7.10 & 6.94 \\
5 & 8.16 & 7.78 \\
\hline$k_{\mathrm{N}}=0.00878 \pm 0.00019 \mathrm{M}^{-1} \mathrm{~s}^{-1}, \quad$ corr. coeff. $=0.9993$
\end{tabular}

Table S42. Kinetic data for the reaction of 4-acetylphenyl benzoate (4d) with morpholine in $80 \mathrm{~mol} \% \mathrm{H}_{2} \mathrm{O} / 20 \mathrm{~mol} \% \mathrm{DMSO}$ at $25.0 \pm 0.1^{\circ} \mathrm{C}$.

\begin{tabular}{ccc}
\hline & {$\left[\mathrm{R}_{2} \mathrm{NH}\right] / 10^{-2} \mathrm{M}$} & $\mathrm{k}_{\mathrm{obsd}} / 10^{-3} \mathrm{~s}^{-1}$ \\
\hline 1 & 4.29 & 1.21 \\
3 & 5.83 & 1.67 \\
4 & 7.10 & 2.07 \\
5 & 8.16 & 2.36 \\
& 9.07 & 2.64
\end{tabular}

$\mathrm{k}_{\mathrm{N}}=0.00299 \pm 0.00003 \mathrm{M}^{-1} \mathrm{~s}^{-1}, \quad$ corr. coeff. $=0.9998$ 
Table 43. Kinetic data for the reaction of 4-(ethoxycarbonyl)phenyl benzoate (4e) with morpholine in $80 \mathrm{~mol} \% \mathrm{H}_{2} \mathrm{O} / 20 \mathrm{~mol} \% \mathrm{DMSO}$ at $25.0 \pm 0.1^{\circ} \mathrm{C}$.

\begin{tabular}{ccc}
\hline & {$\left[\mathrm{R}_{2} \mathrm{NH}\right] / 10^{-1} \mathrm{M}$} & $\mathrm{k}_{\mathrm{obsd}} / 10^{-4} \mathrm{~s}^{-1}$ \\
\hline 1 & 0.812 & 1.17 \\
3 & 0.902 & 1.28 \\
4 & 1.02 & 1.45 \\
5 & 1.28 & 1.77 \\
\hline $\mathrm{k}_{\mathrm{N}}=0.00136 \pm 0.00003 \mathrm{M}^{-1} \mathrm{~s}^{-1}, \quad$ corr. coeff. $=0.9993$
\end{tabular}

